\title{
Minimally invasive versus open radical trachelectomy for early-stage cervical cancer: protocol for a multicenter randomized controlled trial in China
}

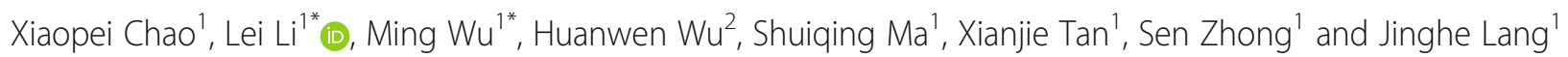

\begin{abstract}
Background: There are limited data comparing the oncologic and fertility outcomes of patients with early-stage cervical cancer (CC) treated by minimally invasive radical trachelectomy (MIRT) or abdominal radical trachelectomy (ART). The purpose of this multicenter study is to compare the oncologic and fertility outcomes of patients treated by MIRT or ART in a randomized controlled manner in China.

Methods: This is a noninferiority, randomized controlled trial performed at 28 Chinese centers; the study is designed to compare the oncologic and fertility outcomes of patients treated by MIRT (robot-assisted or laparoscopic RT) or ART. Patients will be recruited if they have been diagnosed with stage IA1 (with lymphovascular space invasion), IA2, or IB1 CC (with a maximum tumor diameter $\leq 2 \mathrm{~cm}$ ) in the FIGO 2009 staging system and histological subtypes of squamous carcinoma, adenocarcinoma, or adenosquamous carcinoma and if they are also aged 18 to 40 years. These candidates will be randomly assigned to undergo MIRT or ART. The primary endpoint will be disease-free survival. Secondary endpoints will consist of overall and disease-free survival rates, fertility outcomes, and quality of life. A total of 414 patients are needed to accomplish the study goal, with $90.1 \%$ power at a 0.050 significance level to detect an equivalence hazard ratio of 0.75 in the ART group, considering $20 \%$ loss to follow-up.
\end{abstract}

Discussion: The results of the trial should provide robust evidence to surgeons regarding options for the surgical approach in patients with early-stage CC who have a strong willingness to preserve fertility.

Trial registration: ClinicalTrials.gov NCT03739944. Registered on November 14, 2018

Keywords: Cervical cancer, Radical trachelectomy, Progression-free survival, Overall survival, Clinical pregnant rate, Live birth rate, Quality of life, Randomised controlled trial

\footnotetext{
*Correspondence: lileigh@163.com; wuming@pumch.cn

'Department of Obstetrics and Gynecology, Peking Union Medical College Hospital, Shuaifuyuan No. 1, Dongcheng District, Beijing 100730, China

Full list of author information is available at the end of the article
}

(c) The Author(s). 2020 Open Access This article is licensed under a Creative Commons Attribution 4.0 International License, which permits use, sharing, adaptation, distribution and reproduction in any medium or format, as long as you give appropriate credit to the original author(s) and the source, provide a link to the Creative Commons licence, and indicate if changes were made. The images or other third party material in this article are included in the article's Creative Commons licence, unless indicated otherwise in a credit line to the material. If material is not included in the article's Creative Commons licence and your intended use is not permitted by statutory regulation or exceeds the permitted use, you will need to obtain permission directly from the copyright holder. To view a copy of this licence, visit http://creativecommons.org/licenses/by/4.0/ The Creative Commons Public Domain Dedication waiver (http://creativecommons.org/publicdomain/zero/1.0/) applies to the data made available in this article, unless otherwise stated in a credit line to the data. 


\section{Background}

With an estimated 570,000 new cases and 311,000 deaths in 2018 worldwide, cervical cancer (CC) ranks as the fourth most frequently diagnosed cancer and the fourth leading cause of cancer death in women [1]. The majority of CCs occur in low-income and middleincome countries. CC represents a major health challenge in China [2]. The standard surgical procedure for patients with early-stage $\mathrm{CC}$ is radical hysterectomy $(\mathrm{RH})$ and pelvic lymphadenectomy. However, in the last two decades, with the widespread use of CC screening programs, there has been an increase in early-stage CCs diagnosed in women of childbearing age who desire childbearing. Therefore, fertility-sparing surgery (FSS), such as conization and radical trachelectomy (RT), has become one of the options for young women with earlystage $\mathrm{CC}$.

Abdominal radical trachelectomy (ART) was first described by Romanian gynecologist E. Aburel in 1956 [3] and was "rediscovered" by the team of Smith and Ungár in the 1990s [4]. Vaginal RT, first performed by Daniel Dargent, involves resection of the cervix, the upper part of the vagina, and the proximal part of the parametria via a vaginal approach, combined with laparoscopic pelvic lymphadenectomy, while preserving the uterine corpus [5]. However, vaginal dissection in Dargent's procedure must be performed by skilled surgeons. Furthermore, with the progress of minimally invasive technology, minimally invasive RT (MIRT) has allowed for a reduced length of hospital stay and estimated blood loss; decreased analgesic requirements, blood transfusion rates, and complication rates; earlier recovery of physiological functions; and improved esthetic outcomes [6].

A recent multicenter randomized controlled trial (RCT) [7] and a large cohort study [8] of minimally invasive versus abdominal $\mathrm{RH}$ for $\mathrm{CC}$ showed that the minimally invasive surgery (MIS) resulted in decreased survival compared with the laparotomic approach. The results of both studies aroused great controversy over the surgical approaches of CC worldwide. However, few data have been reported to evaluate the oncologic and/ or fertility outcomes of RT with different surgical approaches, except for retrospective studies and systematic reviews. In a previously published paper from our center, ART versus vaginal RT yielded recurrence rates of 0 and $9.8 \%$ [9]. In a systematic review of stage IA to IIA CC, the recurrence rates after ART, laparoscopic RT, and robot-assisted RT were 4.7\% (31/660), 6.3\% (15/238), and $2.2 \%(2 / 89)$, respectively [10]. In another systematic review, among patients with abdominal, vaginal, laparoscopic, and robotic RT, the incidences of cervical stenosis were $11.0 \%, 8.1 \%, 9.3 \%$, and $0 \%$, respectively [11]. The lack of a prospective study promoted the conception of our RCT.

\section{Methods}

\section{Design}

The present investigation is a randomized, controlled, noninferiority, two-arm trial comparing the efficacy of different surgical approaches of RT on the oncology and fertility outcomes in Chinese patients with early-stage CC (Figs. 1 and 2). A total of 28 domestic centers will recruit 414 patients (207 per group) from December 31, 2018, to December 31, 2020. All the surgeries are to be performed by the specialists designated in the research center (the primary investigators are listed in Supplementary Table 1). The patients are randomized on the day of written consent obtainment by the random data website (http:// random.your-data.cn:8095/random), which assigns each patient a randomization number. Specified surgeons from all centers are in charge of enrolling participants. When one potential eligible patient is enrolled, her information will be sent to Dr. LL, who will generate the allocation sequence and assign participants to interventions. The allocation, together with treatment information, will be discussed with the patient by her surgeons when patients attend the clinic to get results, and relevant informed consent will be signed. On the consent form, participants will be asked if they agree to the use of their data should they choose to withdraw from the trial. Participants will also be asked for permission for the research team to share relevant data with people from the universities taking part in the research or from regulatory authorities, where relevant. This trial does not involve collecting biological specimens for storage. Model consent form and other related documentation given to participants and authorized surrogates are available from the corresponding author on request.

Figure 1 refers to the CONSORT flow diagram. Survival outcomes (disease-free survival [DFS] and overall survival $[\mathrm{OS}]$ ) will be analyzed comprehensively, as well as the fertility and pregnancy outcomes and quality of life (QoL).

Any important protocol modifications, happened before or during the trial, will receive a deliberative evaluation by the primary investigator and be approved by the plenary session consisting of all participating centers. Once the modifications were approved, a copy will be sent to the funders and all centers. The clinical trial registry will be updated due to the modifications. Any deviations from the protocol will be fully documented using a breach report form.

\section{Inclusion criteria}

Inclusion criteria are as follows: patients with International Federation of Gynecology and Obstetrics (FIGO) 2009 stage IA1 with lymphovascular space invasion (LVSI), IA2, or IB1 with tumor size $\leq 2 \mathrm{~cm} \mathrm{CC}$; no evidence of upper endocervical involvement (tumor 


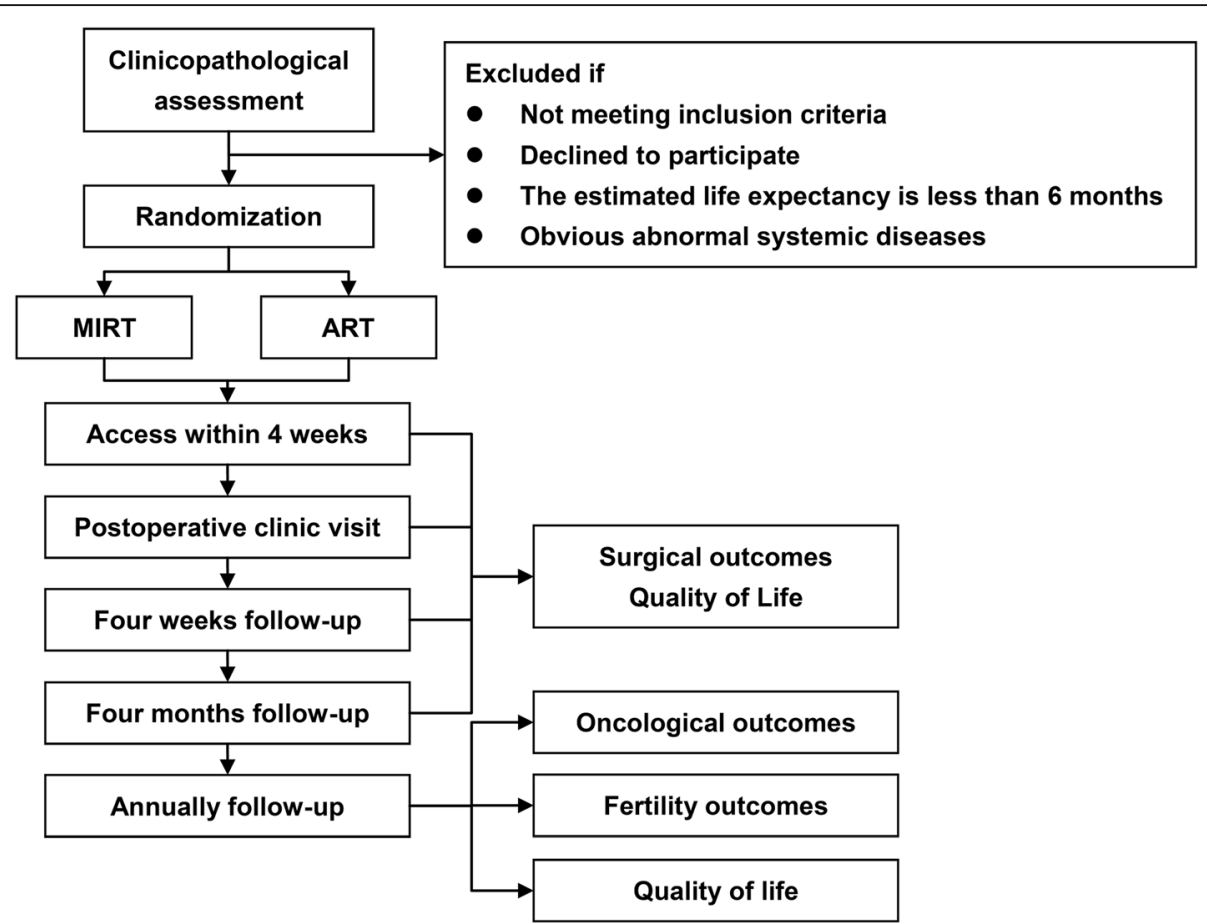

Fig. 1 CONSORT flow diagram of the study. ARH, abdominal radical hysterectomy; MIRT, minimally invasive radical trachelectomy; ART, abdominal radical trachelectomy

confined to the cervix as proved by preoperative imaging, and a cranial extent of the tumor that is at least 1 $\mathrm{cm}$ away from the internal os); no lymph node spread or distant metastasis; histological subtype of squamous cell carcinoma, adenocarcinoma, or adenosquamous carcinoma; aged 18 to 40 years; ability to provide fully informed written consent for participation in this study; strong desire to preserve fertility and no history of fertility impairment; ability to undergo long-term follow-up after surgery; and Eastern Cooperative Oncology Group (ECOG) performance status [12] of 0 to 1.

\section{Exclusion criteria}

The exclusion criteria were as follows: histological subtype of neuroendocrine, clear cell, and serous cell type or metastatic carcinoma; clinically advanced disease (IB1 with tumor size $>2 \mathrm{~cm}$ or stages IB2 to IV); current pregnancy; age younger than 18 or older than 40 years;

\begin{tabular}{|c|c|c|c|c|c|c|c|c|}
\hline \multirow[b]{3}{*}{ Time point } & \multicolumn{8}{|c|}{ Study period } \\
\hline & \multirow[t]{2}{*}{ Enrollment } & \multirow{2}{*}{$\begin{array}{c}\text { Allocation } \\
0\end{array}$} & \multicolumn{5}{|c|}{ Postallocation } & \multirow[t]{2}{*}{ Closeou } \\
\hline & & & $\begin{array}{l}\text { Within } 4 \text { weeks } \\
\text { before surgery }\end{array}$ & $\begin{array}{l}\text { Postoperative } \\
\text { clinic visit }\end{array}$ & $\begin{array}{l}\text { Four weeks } \\
\text { follow-up }\end{array}$ & $\begin{array}{c}\text { Four months } \\
\text { follow-up }\end{array}$ & $\begin{array}{l}\text { Annually } \\
\text { follow-up }\end{array}$ & \\
\hline \multirow{4}{*}{$\begin{array}{l}\text { Enrollment: } \\
\text { Eligibility screen } \\
\text { Informed consents } \\
\text { Allocation }\end{array}$} & & & & & & & & \\
\hline & $\mathrm{x}$ & & & & & & & \\
\hline & $x$ & & & & & & & \\
\hline & & $\mathrm{x}$ & & & & & & \\
\hline \multirow{2}{*}{$\begin{array}{l}\text { Intervention: } \\
\text { Radical hysterectomy }\end{array}$} & & & & & & & & \\
\hline & & $\mathrm{x}$ & & & & & & \\
\hline \multirow{5}{*}{$\begin{array}{l}\text { Assessment: } \\
\text { Surgical outcomes } \\
\text { Quality of life } \\
\text { Oncological outcomes } \\
\text { Fertility outcomes }\end{array}$} & & & & & & & & \\
\hline & & & & 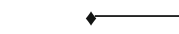 & & & $\longrightarrow$ & \\
\hline & & & $\mathrm{x}$ & $\mathrm{x}$ & $x$ & $\mathrm{x}$ & $\mathrm{x}$ & \\
\hline & & & & $\leftrightarrow$ & & & - & $\mathrm{x}$ \\
\hline & & & & $\leftarrow$ & & & $\rightarrow$ & $\mathrm{x}$ \\
\hline
\end{tabular}

Fig. 2 SPIRIT figure of the study 
having received neoadjuvant chemotherapy or radiotherapy; without a systematic preoperative imaging evaluation (pelvic MRI with or without PET/CT); unwillingness to participate in this study; and preexisting malignancy, severe systemic disease or severe mental illness.

\section{Main objective and primary endpoint}

The primary objective of the analysis is to evaluate the 5-year DFS related to different surgical approaches for radical trachelectomy (RT). The primary endpoint will be recurrence confirmed by imaging or histology.

\section{Secondary objectives and secondary endpoints}

The secondary objectives are as follows:

1. To evaluate the OS at the 5-year follow-up

2. To assess different strategies of RT in terms of fertility and obstetric outcomes

3. To compare the incidence of perioperative complications of different surgical approaches

4. To compare major perioperative complications within 28 days after surgery and major postoperative complications

5. To determine the general and specific QoL

Secondary endpoints will therefore be the following:

1. All-cause mortality

2. Fertility outcomes consisting of cervical incompetence, natural pregnancy, and infertility treatment such as intrauterine insemination or IVFET; obstetric outcomes consisting of the time interval between the RT surgery and pregnancy; the overall mean length of time during which conception was attempted; the number of pregnancies; the mode of delivery; and the outcome of each pregnancy, such as spontaneous abortion, induced abortion, drug abortion, preterm birth, premature rupture of membranes, fetal growth restriction, and full-term birth

3. Perioperative complications consisting of injury of the ureter, bladder, bowel, blood vessels, or nerves, and vaginal bleeding, and postoperative complications consisting of postoperative pelvic infection, pelvic lymphocele, intrauterine infection, Asherman syndrome, abnormal menstruation, urinary tract infection, hydronephrosis, intestinal obstruction, chronic pain, varices at the site of uterovaginal anastomosis, and fistula formation

4. Major perioperative and postoperative complications consisting of early postoperative complications (cerebrovascular, pulmonary, and renal diseases; ileus; abdominal wound complications; septicemia; thromboembolism; and lymphocele formation and secondary infection) and delayed postoperative complications (lymphedema, vaginal evisceration)

5. QoL assessment comprising the authorized Chinese edition of EORTC QLQ-C30 [13] and its CC module QLQ-CX24 [14], the 19-item Female Sexual Function Index (FSFI) [15], and the PFIQ-7 questionnaire [16].

\section{Description of the parameters for assessing efficacy endpoints}

The primary endpoint is a composite endpoint, which will be collected at each visit. Surgical complications will be assessed by surgeons and/or a specialist if he or she has been consulted for a complication. Obviously, a double-blinded trial was not feasible, i.e., the surgeons and clinicians managing the patients have to explain the operation method and risks to the patients, and in turn, the patients must sign their informed consent. However, the information of patients and surgeons are blinded to performers dealing with data analysis.

\section{Learning curve}

In this study, all surgeons have been qualified by the existing continuous clinical data and survival outcomes of at least 10 patients with early-stage CC who have undergone RT, including at least 5 cases of MIRT. Meanwhile, the surgeons should provide all surgical reports and at least two integrated copies of surgical videos as the competence of these surgeries. These data and videos will be presented as supplementary material for the final report. The survival outcomes are used to assess the learning curves of participating surgeons, and the videos are used to assess the surgical skills.

\section{Surgical treatment}

Patients with early-stage CC who are eligible for enrollment will be randomly assigned to the MIRT group or ART group. Evaluation of the pelvic nodes in patients with early-stage $\mathrm{CC}$ will be performed. The pelvic lymph nodes may be assessed with a complete lymphadenectomy or via sentinel lymph node mapping. Surgical details, including uterine artery preservation, the cerclage material and position of knot placement, and the method to prevent cervical stenosis, will be recorded in the surgical records.

\section{ART}

Entry into the abdomen may be accomplished via a midline vertical incision to gain adequate access to the pelvis. After excising the pelvic lymph nodes, the paravesical and pararectal spaces are developed. The round ligaments are grasped with Kelly clamps for 
uterine manipulation, and the vesicocervical space is developed to dissect the bladder off the cervix. Care is taken to preserve the ovary pelvic funnel ligaments and utero-ovarian vessels, as these are the main blood supply to the remaining uterus. The uterine vessels are preserved, while their descending branches are resected at the level of the isthmus. Once complete ureterolysis is performed to the tunnel of Wertheim, the uterine vessels are divided at their origin from the hypogastric vessels. The parametria and paracolpos are mobilized with the trachelectomy specimen. The posterior cul-de-sac is incised, and the uterosacral ligaments are divided at their origin. The vagina is then incised to perform an anterior colpotomy $1-2 \mathrm{~cm}$ distal to the external cervical os. This incision is then carried circumferentially until the specimen is completely separated from the vagina. The lower uterine segment is estimated, and clamps are placed at the level of the internal os. The cervix is then incised with a knife approximately $5 \mathrm{~mm}$ below the internal os. Patency of the remaining cervical canal may be achieved with the use of a Foley or Malecot catheter followed by the placement of an endocervical cerclage. The lower uterine segment is then sutured to the vaginal mucosa via interrupted or continuous sutures [17].

\section{MIRT}

The procedure with either a laparoscopic or robotic approach is the same with regard to the abdominal approach. A uterine manipulator must be avoided. A vaginal excision must be made under vaginal exposure rather than under peritoneal exposure.

\section{Safety and adverse events}

The adverse events and their severity will be judged by the criteria of the Common Terminology Criteria for Adverse Events [18]. This trial will be conducted in compliance with this study protocol.

\section{Data management}

All medical information required according to the protocol will be collected via the case report forms based on the website, facilitating the real-time, central assessment of the data completeness and patient follow-up. This information will include demographic data, the surgical approach, the skin-to-skin operative time, EBL, and the surgical energy devices. The pathogenic material will also be collected, including the results regarding the tumor histology subtype, FIGO stage, grade (well, moderately or poorly differentiated), tumor size, depth of stromal invasion, LVSI, lymph node status, and surgical margin status. After RT, data on surgical complications, bladder/intestinal function recovery, QoL, fertility and obstetrics outcomes, and disease recurrence and survival information will also be followed.
The questionnaires will be completed, the ovarian reserve function will be evaluated, and a urodynamic study (UDS) and anorectal manometry will be assessed within 4 weeks before surgery, at 4 months postoperatively, at 12 months postoperatively, and then once a year thereafter. After the RT (Table 1), the patients will be required to attend the customed follow-up regularly, at 3 - to 6-month intervals in the first 2 years after completing the treatment, 6- to 12-month intervals from the third to the fifth year after treatment, and then at 1-year intervals thereafter. The effects of the follow-up scheme (pelvic examination, the ThinPrep cytological test and/or human papillomavirus testing, transvaginal ultrasonography, an abdominal ultrasound scan, chest radiography, and serum tumor marker, i.e., squamous cell carcinoma and Ca125) will be evaluated at each visit.

This trial will be conducted in compliance with the current version of the protocol, with all statutory and regulatory requirements. The Data Safety Monitoring Committee (Supplementary Table 2) will regularly receive study data, including complications, patient conditions, and survival outcomes. This committee will be composed of physicians, administration staff, and scientific researchers, who are not participating in the study as investigators and who will be provided with available collected data during the study. The committee reserves the right to terminate the study at any time for medical or administrative reasons. In the case of loss to followup, the surgeons or investigators will do their best to contact each patient involved to identify if he/she is alive. If an individual leaves the research study prematurely, data related to the participant can still be used unless an objection was recorded when the patient signed the consent form. If consent is withdrawn, no data about the individual may be used unless the patient states in writing that he/she does not object.

For participants who consent to participate the trial but deviate from intervention protocols, i.e., refuse the randomized allocation, the surgical and follow-up data will still be collected the same as participants following the protocol, which will be analyzed according to intention to treat.

The trial results will be communicated to the funders, investigators, and other relevant groups via publications, reporting results in databases, data sharing arrangements, and social media (WeChat).

\section{Description of statistical methods Sample size calculation}

Systematic reviews have revealed that the rate of disease recurrence after ART is 3.8 to $4.7 \%[10,19]$. The 5 -year DFS of ART in a Chinese study was 96.5\% [20]. In our study, we assume that the recurrent rate of ART of 5 years is $5 \%$. A noninferiority log-rank test with an overall 
Table 1 Lists of clinical assessments at different time points

\begin{tabular}{|c|c|c|c|}
\hline Check-up time & Inspection items & Documentors & Directors \\
\hline \multirow[t]{5}{*}{ Within 4 weeks before RT } & Urodynamic assessment & CRF & Research centers \\
\hline & Anorectal manometry & CRF & \\
\hline & QoL assessment & CRF & \\
\hline & Sexual function scale & CRF & \\
\hline & Ovarian reserve function & CRF & \\
\hline \multirow[t]{3}{*}{ Ten to 14 days after RT } & Operation details & CRF & Research centers \\
\hline & Residual urine & CRF & \\
\hline & Perioperative complications & CRF & \\
\hline \multirow[t]{2}{*}{ Four weeks after RT } & Postoperative complications & CRF & \\
\hline & Pathological report & CRF & \\
\hline \multirow[t]{6}{*}{ Four months after RT } & Urodynamic assessment & CRF & Research centers \\
\hline & Anorectal manometry & CRF & \\
\hline & QoL assessment & CRF & \\
\hline & Ovarian reserve function & CRF & \\
\hline & Postoperative adjuvant therapy & CRF & \\
\hline & Data curation & Database & Main center \\
\hline \multirow[t]{9}{*}{ Every 12 months after RT } & Urodynamic assessment & CRF & Research centers \\
\hline & Anorectal manometry & CRF & \\
\hline & QoL assessment & CRF & \\
\hline & Sexual function scale & CRF & \\
\hline & Ovarian reserve function & CRF & \\
\hline & Pregnant desires and outcomes & CRF & \\
\hline & Survival outcome & CRF & \\
\hline & Data curation & Database & Main center \\
\hline & Follow-up time, scheme, and results & Database & \\
\hline
\end{tabular}

CRF case report form, QoL quality of life, $R T$ radical trachelectomy

sample size of 331 subjects (165 in the reference group and 166 in the treatment group) achieves $90.1 \%$ power at a 0.050 significance level to detect an equivalence hazard ratio of 0.75 when the actual hazard ratio is an equivalence hazard ratio of 1.00 , and recurrence rates of 5 years in the MIRT and ART group are 7 and 5\% [21]. Considering the possible $20 \%$ rate of loss to follow-up, 414 patients are needed to accomplish the study goal.

\section{Statistics}

Continuous variables conformed to the normal distribution will be described with means and standard deviations, and discrete variables not conformed to the normal distribution will be summarized with medians, ranges, and interquartile ranges. The $t$ test will be used for continuous variables, and the chi-square test or Fisher's exact test will be used for categorical variables. To evaluate the strength of associations, bivariate and multivariable logistic regression analyses will be used, and the strength of associations will be expressed as hazard ratios (HR) with 95\% confidence intervals (95\% CI).
Kaplan-Meier plots will be generated for recurrence and death rates between the groups, and the log-rank test will be applied for the eventual significant differences. The survival outcomes will be compared according to intention to treat and per protocol. The analysis of intention to treat is available for protocol nonadherence. These statistical analyses will be performed using SPSS 22.0 (SPSS Inc., Chicago, IL, USA). All analyses will be two-sided, and significance will be set at a $P$ value of 0.05 .

\section{Strength and limitations of this study}

To our knowledge, this is the first multicenter RCT designed for the comparison of oncologic and fertility outcomes of different surgical routes of RT, despite a registered retrospective multicenter trial [22].

With the advantage of sufficient patients with earlystage CC in the study centers, this study can be implemented as soon as possible. 
This study takes the surgeon as one of the important parameters and as the main consideration, which significantly differs from the LACC trial [7]. For the first time, the learning curve for MIS will be taken into the survival analysis.

This study will make uniform reports of the pathological outcomes. The comprehensive and meticulous pathological data will support the information about the prognosis.

The emphasis on the individual surgeon's experience and skill will most likely limit generalization.

This study will not evaluate the type of suture material used to perform the cerclage, such as braided and nonbraided sutures, or the position of the knot placement and the time of cerclage removal before delivery.

This RCT will explore the effect of different methods to prevent cervical stenosis, such as a Nelaton catheter, an intrauterine device, and a Smit sleeve, as well as the time of removal of these materials.

\section{Discussion}

In our study, we have enrolled women younger than 40 years, with stage IA1 disease with LVSI, stage IA2, and smaller stage IB1 tumors ( $\leq 2 \mathrm{~cm}$ diameter); without evidence of lymph node metastases on imaging; with evidence of endocervical extension on MRI; and with a desire to preserve fertility. At present, five different FSS procedures are available for patients with $\mathrm{CC}$ : conization, a simple trachelectomy, VRT, MIRT (including laparoscopic and robot-assisted routes), ART, extraperitoneal RT (a novel fertility-preserving option) [23], and neoadjuvant chemotherapy (NACT) followed by FSS (conization/simple trachelectomy or RT). MIS has gained wide acceptance secondary to the implied advantages of a faster return to normal activity and diet, reduced hospital stay and postoperative discomfort, and decreased scarring and rate of adhesive. Although the open approach is more radical in terms of parametrial and paracervical resection [10], it has theoretical drawbacks, particularly the formation of adhesions and scar tissue that may ultimately impact a woman's fertility, increased blood loss, and a prolonged hospital stay.

Additionally, with the advent of the sentinel lymph node procedure, the morbidity in this usually young patient population has continued to improve. There are adequate data to prove that oncologic outcomes, concerning recurrence and mortality, are comparable to those of $\mathrm{RH}$ and that the obstetrical outcomes are favorable [17]. However, a recent study has put forward contrasting conclusions; thus, we aim to evaluate the impact of different surgical approaches of RT on survival and obstetric outcomes.
One of the crucial designs is the consideration of the learning curves of surgeons. A learning curve is defined by the number of surgical procedures performed by a surgeon before he or she reaches an accepted plateau in objective outcomes such as operative time, estimated blood loss (EBL), complication rate, and surgical performance [24]. Research defining learning curves in gynecologic oncology surgery is limited but has shown that gynecologic oncology surgeons can become proficient after 20 cases of robotic hysterectomy with pelvic-aortic lymph node dissection and that their performance can be continually improved in the period between 50 and 70 cases [25]. Compared with vaginal RT, ART has a shorter learning curve, requires minimal additional training, and has a higher oncological radicality. ART is similar to the abdominal $\mathrm{RH}$ procedure that most gynecologic oncologists are familiar with [26-28]. This suggests that experience is critical in laparoscopic and robotic procedures [29]. However, there is little literature about the learning curve for MIRT. Therefore, each study center would appoint only one specific expert who will manage all the major procedures of RT, and their quality would be evaluated by the amount and survival outcomes of the surgeries.

\section{Trial status}

Recruitment of participants started in December 2018, and the last participant is expected to reach the primary endpoint (5-year follow-up) in December 2025. Primary data analysis will begin in December 2023. The naturalistic follow-up phase of the trial will continue until December 2025. The protocol version number and date were 3.0 and December 23, 2018, respectively.

\section{Supplementary Information}

The online version contains supplementary material available at https://doi. org/10.1186/s13063-020-04938-3.

Additional file 1: Supplementary Table 1. Study centers and their primary investigators, contact staff and phone numbers.

Additional file 2: Supplementary Table 2. Members of the Data Safety Monitoring Committee.

\footnotetext{
Abbreviations

MIRT: Minimally invasive radical trachelectomy; ART: Abdominal radical trachelectomy; CC: Cervical cancer; FSS: Fertility-sparing surgery; RT: Radical trachelectomy; MIS: Minimally invasive surgery; RCT: Randomized controlled trial; DFS: Disease-free survival; OS: Overall survival; LVSI: Lymphovascular space invasion; QoL: Quality of life; NSRT: Nerve-sparing radical trachelectomy; EBL: Estimated blood loss; LQ: Leiden Questionnaire; UDS: Urodynamic study; PVR: Postvoid residual volume; MFR: Maximum flow rate; AFR: Average flow rate; MUCP: Maximal urethral closure pressure; FSFI: Female Sexual Function Index; UIQ-7: Urinary Impact Questionnaire; CRAIQ-7: Colorectal-Anal Impact Questionnaire; POPIQ-7: Pelvic Organ Prolapse Impact Questionnaire; HR: Hazard ratios; CTA: Computed tomography angiography; NACT: Neoadjuvant chemotherapy
} 


\section{Acknowledgements}

None.

\section{Statement of submission}

The paper is not under consideration by another journal, and the results presented in this work have not been previously presented or published.

\section{Authors' contributions}

$\mathrm{LL}$ and MW conceived the study, designed the work, and drafted the manuscript. XC designed the work and drafted the manuscript. SM, XT, SZ, and HW designed the work. HW conducted the pathological evaluation. All authors have approved the final version of the manuscript. All named authors adhere to the authorship guidelines of Trials. All authors have agreed to publication. No professional writers have been involved.

\section{Funding}

This study is supported by the Chinese Academy of Medical Sciences Initiative for Innovative Medicine (CAMS-2017-I2M-1-002). The funder confirmed funding for the trial. However, the funders have had no role in the study design, data collection and analysis, decision to publish, or preparation of the manuscript.

\section{Availability of data and materials}

The datasets generated and/or analyzed during the current study are available in the public repository, http://117.50.88.118, and are also available from the corresponding author on reasonable request.

\section{Ethics approval and consent to participate}

The Institutional Review Board of Peking Union Medical College Hospital has approved this study (No. JS-1712), and we will not begin recruiting at other centers in the trial until local ethical approval has been obtained. The registration number is NCT03739944 (ClinicalTrials.gov). All patients have provided their consent to participate in the study before enrollment.

As staff from the central study center, we confirmed our study has gained ethical approval from all participating centers.

This study has been approved by the Institutional Review Board of Peking Union Medical College Hospital (registration no. JS-1712). All procedures are in accordance with the ethical standards of the responsible institutional and national committee on human experimentation and with the Declaration of Helsinki of 1964 and its later amendments. Written informed consent will be obtained from all eligible participants before enrollment. This study does not involve animal subjects.

\section{Consent for publication}

Not applicable.

\section{Competing interests}

All authors declare that they have no conflicts of interest to disclose.

\section{Author details}

'Department of Obstetrics and Gynecology, Peking Union Medical College Hospital, Shuaifuyuan No. 1, Dongcheng District, Beijing 100730, China. ${ }^{2}$ Department of Pathology, Peking Union Medical College Hospital, Beijing 100730, China

Received: 1 November 2019 Accepted: 27 November 2020

Published online: 14 December 2020

\section{References}

1. Bray F, Ferlay J, Soerjomataram I, Siegel RL, Torre LA, Jemal A. Global cancer statistics 2018: GLOBOCAN estimates of incidence and mortality worldwide for 36 cancers in 185 countries. CA Cancer J Clin. 2018;68:394-424.

2. Chen W, Zheng R, Baade PD, Zhang S, Zeng H, Bray F, et al. Cancer statistics in China, 2015. CA Cancer J Clin. 2016;66:115-32

3. Capilna ME, Szabo B, Becsi J, loanid N, Moldovan B. Radical trachelectomy performed during pregnancy: a review of the literature. Int J Gynecol Cancer. 2016;26:758-62.

4. Smith JR, Boyle DC, Corless DJ, Ungar L, Lawson AD, Del Priore G, et al. Abdominal radical trachelectomy: a new surgical technique for the conservative management of cervical carcinoma. Br J Obstet Gynaecol. 1997;104:1196-200.
5. Dargent $D$, Martin $X$, Sacchetoni $A$, Mathevet $P$. Laparoscopic vaginal radical trachelectomy: a treatment to preserve the fertility of cervical carcinoma patients. Cancer. 2000;88:1877-82.

6. Obermair A, Gebski V, Frumovitz M, Soliman PT, Schmeler KM, Levenback C, et al. A phase III randomized clinical trial comparing laparoscopic or robotic radical hysterectomy with abdominal radical hysterectomy in patients with early stage cervical cancer. J Minim Invasive Gynecol. 2008;15:584-8.

7. Ramirez PT, Frumovitz M, Pareja R, Lopez A, Vieira M, Ribeiro R, et al. Minimally invasive versus abdominal radical hysterectomy for cervical cancer. N Engl J Med. 2018;379:1895-904.

8. Melamed A, Margul DJ, Chen L, Keating NL, Del Carmen MG, Yang J, et al. Survival after minimally invasive radical hysterectomy for early-stage cervical cancer. N Engl J Med. 2018;379:1905-14.

9. Cao DY, Yang JX, Wu XH, Chen YL, Li L, Liu KJ, et al. Comparisons of vaginal and abdominal radical trachelectomy for early-stage cervical cancer: preliminary results of a multi-center research in China. Br J Cancer. 2013;109: 2778-82.

10. Bentivegna E, Gouy S, Maulard A, Chargari C, Leary A, Morice P. Oncological outcomes after fertility-sparing surgery for cervical cancer: a systematic review. Lancet Oncol. 2016;17:e240-e53.

11. Li X, Li J, Wu X. Incidence, risk factors and treatment of cervical stenosis after radical trachelectomy: a systematic review. Eur J Cancer. 2015;51:1751-9.

12. Ruckdeschel JC, Finkelstein DM, Ettinger DS, Creech RH, Mason BA, Joss RA, et al. A randomized trial of the four most active regimens for metastatic non-small-cell lung cancer. J Clin Oncol. 1986;4:14-22.

13. Greimel ER, Kuljanic Vlasic K, Waldenstrom AC, Duric VM, Jensen PT, Singe $S$, et al. The European Organization for Research and Treatment of Cancer (EORTC) quality-of-life questionnaire cervical cancer module: EORTC QLQCX24. Cancer. 2006;107:1812-22.

14. Hua CH, Guo HM, Guan XL, Kong FJ, Hou RJ, Zhang XY, et al. Validation of the European Organization for Research and Treatment of Cancer cervical cancer module for Chinese patients with cervical cancer. Patient Prefer Adherence. 2013;7:1061-6.

15. ter Kuile MM, Brauer M, Laan E. The Female Sexual Function Index (FSFI) and the Female Sexual Distress Scale (FSDS): psychometric properties within a Dutch population. J Sex Marital Ther. 2006:32:289-304.

16. Swift S, Woodman P, O'Boyle A, Kahn M, Valley M, Bland D, et al. Pelvic Organ Support Study (POSST): the distribution, clinical definition, and epidemiologic condition of pelvic organ support defects. Am J Obstet Gynecol. 2005;192:795-806.

17. Costales A, Michener C, Escobar-Rodriguez PF. Radical trachelectomy for early stage cervical cancer. Curr Treat Options in Oncol. 2018;19:75.

18. Common Terminology Criteria for Adverse Events (CTCAE) v4.03 (https:// ctep.cancer.gov/protocolDevelopment/electronic_applications/ctc. htm\#ctc_50). National Cancer Institute.

19. Pareja R, Rendon GJ, Sanz-Lomana CM, Monzon O, Ramirez PT. Surgical, oncological, and obstetrical outcomes after abdominal radical trachelectomy - a systematic literature review. Gynecol Oncol. 2013;131:7782.

20. Li X, Li J, Wen H, Ju X, Chen X, Xia L, et al. The survival rate and surgical morbidity of abdominal radical trachelectomy versus abdominal radical hysterectomy for stage IB1 cervical cancer. Ann Surg Oncol. 2016;23:2953-8.

21. Lakatos E. Sample sizes based on the log-rank statistic in complex clinical trials. Biometrics. 1988:44:229-41.

22. Salvo G, Ramirez PT, Leitao M, Cibula D, Fotopoulou C, Kucukmetin A, et al. International radical trachelectomy assessment: IRTA study. Int J Gynecol Cancer. 2019;29:635-8.

23. Mabuchi S, Kimura T. Extraperitoneal radical trachelectomy with pelvic lymphadenectomy: a novel fertility-preserving option for early stage cervical cancer patients. Int J Gynecol Cancer. 2017;27:537-42.

24. Schreuder HW, Verheijen RH. Robotic surgery. BJOG. 2009;116:198-213.

25. Seamon LG, Fowler JM, Richardson DL, Carlson MJ, Valmadre S, Phillips GS, et al. A detailed analysis of the learning curve: robotic hysterectomy and pelvic-aortic lymphadenectomy for endometrial cancer. Gynecol Oncol. 2009;114:162-7

26. Wethington SL, Cibula D, Duska LR, Garrett L, Kim CH, Chi DS, et al. An international series on abdominal radical trachelectomy: 101 patients and 28 pregnancies. Int J Gynecol Cancer. 2012;22:1251-7.

27. Ungar L, Palfalvi L, Hogg R, Siklos P, Boyle DC, Del Priore G, et al. Abdominal radical trachelectomy: a fertility-preserving option for women with early cervical cancer. BJOG. 2005;112:366-9. 
28. Capilna ME, loanid N, Scripcariu V, Gavrilescu MM, Szabo B. Abdominal radical trachelectomy: a romanian series. Int J Gynecol Cancer. 2014;24: 615-9.

29. Evans JM, Karram MM, Mahdy A, Robertshaw D. Urinary tract injury at the time of laparoscopic and robotic surgery: presentation and management. Female Pelvic Med Reconstr Surg. 2013;19:249-52.

\section{Publisher's Note}

Springer Nature remains neutral with regard to jurisdictional claims in published maps and institutional affiliations.

Ready to submit your research? Choose BMC and benefit from:

- fast, convenient online submission

- thorough peer review by experienced researchers in your field

- rapid publication on acceptance

- support for research data, including large and complex data types

- gold Open Access which fosters wider collaboration and increased citations

- maximum visibility for your research: over $100 \mathrm{M}$ website views per year

At BMC, research is always in progress.

Learn more biomedcentral.com/submissions 